\title{
Static, Vibration Analysis and Sensitivity Analysis of Stepped Beams Using Singularity Functions
}

\author{
Peng Cheng, Carla Davila, and Gene Hou \\ Department of Mechanical and Aerospace Engineering, Old Dominion University, Norfolk, VA 23529, USA \\ Correspondence should be addressed to Gene Hou; ghou@odu.edu
}

Received 30 September 2013; Accepted 5 December 2013; Published 20 January 2014

Academic Editor: Yiyang Li

Copyright (C) 2014 Peng Cheng et al. This is an open access article distributed under the Creative Commons Attribution License, which permits unrestricted use, distribution, and reproduction in any medium, provided the original work is properly cited.

\begin{abstract}
A systematic approach is presented in this paper to derive the analytical deflection function of a stepped beam using singularity functions. The discontinuities considered in this development are associated with the jumps in the flexural rigidity and the applied loads. This approach is applied to static and vibration analyses of stepped beams. The same approach is later extended to perform sensitivity analysis of stepped beams. This is done by directly differentiating the analytical deflection function with respect to any beam-related design variable. The particular design variable considered here is the location of discontinuity in flexural rigidity. Example problems are presented in this paper to demonstrate and verify the derivation process.
\end{abstract}

\section{Introduction}

The stepped beams can be found in many engineering applications in shafts, antennae, rotor blades, gun barrels, slender structures, and so forth. The changes in the cross-sectional areas and the load distribution generate discontinuities in deriving the deflection equation of a stepped beam. Many have introduced singularity or Macaulay functions to handle these discontinuities. The singularity or Macaulay functions, made of the Dirac delta function and its derivatives, can be rigorously defined based upon theories of distributions or generalized functions [1-3].

The goal of this paper is to develop a unified, singularity function-based approach to perform static, vibration, and associated design sensitivity analysis of stepped beam problems. The approach is developed based upon an observation that the product of the bending moment and the inverse of the moment of inertia can be spanned as the algebraic sum of terms. Each of these terms contains only one singularity function of zero or higher order. These terms constitute the right-hand side of the bending moment equation of the stepped beam, which can be easily integrated to obtain the analytical expression of deflection. Such analytical function can be conveniently differentiated to obtain the analytical expression of sensitivity of beam deflection with respect to any design variables. Sensitivity analysis aims to find the derivatives of structural responses, such as deflection and frequencies, with respect to structural-related parameters. Sensitivity analysis has broad applications in support of reanalysis, design optimization, and reliability analysis $[4,5]$. For example, the effects of structural degrading, crack propagation, and local damage of a stepped beam can be effectively estimated through the use of the sensitivity equations derived in this paper.

Falsone [6] used the singularity functions to find the analytical expression of the deflection of an Euler-Bernoulli beam with a uniform cross-section, subjected to discontinuities in the loads, deflections, and slopes. These discontinuities are expressed in terms of the Heaviside function and the Dirac delta function, as well as their derivatives and integrals. They appear as the right-hand side terms in the 4th order differential equation of the deflection. Yavari and his colleagues $[7,8]$ provided a mathematical foundation for the distributional derivatives of the Dirac delta function based upon the Schwarz's distribution theory. With this foundation, they derived the Euler-Bernoulli and the Timoshenko beam bending equations in terms of generalized functions. The discontinuities they considered in flexible stiffness, slope, and deflection are resulted from the change in moment of inertia and the point supports of internal linear and torsional springs. 
Notice that Yavari and his colleagues started their derivation of the governing differential equation by piecing the beam deflections of uniform sections together with the use of Heaviside function. The beam deflection is then differentiated according to the theories of distributions up to the 4 th order. In the process, the jump conditions in deflection, slope, moment, and shear force are converted into equivalent load distributions. The resultant 4 th order beam equation can then be solved with the help of an auxiliary beam equation, which is established to solve the distribution part of the deflection equation. The unknown amounts of jumps in deflection and slope appear as part of the forcing term in the final beam equation, which can be solved based upon the continuous conditions at the jump point.

Biondi and Caddemi [9] expressed the moment of inertia distribution in terms of a Dirac delta and a Heaviside function to count the discontinuities in beam's flexibility and slope. The forcing term of the beam equation includes the products of the deflection, its derivatives, and the Dirac delta function. The beam equation is then integrated to obtain the shear force, moment, slope, and deflection. The intensity of the discontinuity of moment of inertia can be determined based upon the jump in the slope. The process involves only four integration constants which are determined by the boundary conditions. However, the process requires establishing four auxiliary beam equations. Later, Biondi and Caddemi [10] extended their method to find the deflections of stepped Euler beams and planar frames with internal hinges and rotational springs. A special integration equation was employed to compute the product of two Dirac's deltas at the same location. Palmeri and Cicirello [11] used distribution Daric delta functions to model the slope discontinuity caused by a crack in the uniform beam. In their study, the distribution functions are applied to model the reciprocal of the moment of inertia, rather than the moment of inertia itself. Other singularity function-like methods have been introduced in the literature to solve stepped beam problems, such as close-form finite element method [12] and the discrete singular convolution [13]. The latter, however, are approximated methods.

Vibration analysis of a stepped beam also attracts much research interest. The resultant natural frequencies are used to study the rotatory resonance of a shaft that carries gears as additional weights. Naguleswaran [14] used the eigenfunction of an Euler-Bernoulli beam to represent the mode shape of each uniform section of the stepped beam studied in his work. The conditions that the eigenfunction and the associated slope, moment, and shear force are continuous at the junction of the change in the cross-section are used to connect the eigenfunction of one uniform beam with the other. The author later extended the method to solve vibration problems of beams with multiple steps and various support conditions [15].

Mann and his colleagues $[16,17]$ followed similar strategy discussed in $[14,15]$ to solve the vibration of cantilever stepped beams. The boundary conditions of the cantilever beam allow the mode shapes of a uniform section of the beam to be determined one after another from the fixed to the free end, based upon the continuous conditions at the intersections. Experimental validation was provided in their paper to validate the solutions.

The Rayleigh-Ritz method was used by Popplewell and Chang [18] to find the eigensolution of a stepped beam. The trial functions included the eigenfunctions of a uniform beam and the "force mode" functions. The force mode functions are the static deflection of a uniform beam subjected to a unit force or moment applied at the junction of changes in beam's sectional properties. They are in fact the influence coefficients used in the current study. Their results were compared favorable to the exact solutions.

Lu et al. [19] developed a finite element method called composite element model (CEM) to perform vibration analysis of multiple-stepped beams. In addition to the commonly used shape functions, the authors add the globally defined analytical expressions resulted from the classical beam theory as part of the trial functions. The CEM can be viewed as an extension of the Rayleigh-Ritz method [18]. Use of the singularity functions for vibration analysis of stepped beams was not the focus of the research in [19].

Only limited works are found to be related to design sensitivity analysis and optimization of stepped beams. Naguleswaran [14] approximated sensitivity coefficients of eigenvalues by finite differencing. The design variables considered are the relative ratios between the lengths, masses, and moments of inertia of uniform sections with different sectional properties. Lepik [20] introduced a cubic function to smoothen the jump in the beam's dimension. This approximation enabled the classical optimization methods to be used to find the dimensions of a stepped beam that minimizes its dynamic responses subjected to a constant volume constraint.

Organization of this paper is described below. Formulas that span the product of singularity functions are summarized in Section 2. The Euler-Bernoulli theory is used in this study to describe the deflection of the stepped beam. The example problems studied here include a simply supported beam, a continuous beam with a roller support, and a continuous beam with a linear spring support. These example beams are subjected to discontinuities in flexural rigidity and loads. The discontinuity in flexural rigidity, referred later in the paper as rigidity discontinuity, is caused by change in the cross-sectional area. The static analysis and vibration analysis of these example beams are given in Sections 3 and 4 , respectively. The sensitivity analyses of the static deflection and the eigenvalues with respect to the location of discontinuity in flexural rigidity are investigated in Section 5. Only the simply supported stepped beam is considered as an example in Section 5 for sensitivity analysis. The results obtained by the proposed approach will be compared with those obtained by the commercially rated finite element code, MD NASTRAN. Notations of singularity functions used in this paper follow those defined in [3]. The conclusion remarks are given in the final section, Section 6 .

\section{Product of Singularity Function}

Singularity functions or singularity brackets are used to describe the discontinuous functions. The bracket, $\langle x-a\rangle^{n}$, 
used in [3] for an integer $n$ will be used here to represent a singularity function with some level of discontinuity at $a$. A singularity function with a positive integer $n, n>0$, is defined as

$$
\begin{gathered}
\langle x-a\rangle^{n}= \begin{cases}0 & \text { if } x<a \\
(x-a)^{n} & \text { if } x \geq a\end{cases} \\
\int_{-\infty}^{x}\langle x-a\rangle^{n} d x=\frac{\langle x-a\rangle^{n+1}}{n+1} .
\end{gathered}
$$

The singularity function with $n=0$ is the Heaviside function defined as

$$
\langle x-a\rangle^{0}= \begin{cases}0 & \text { if } x<a \\ \frac{1}{2} & \text { if } x=a \\ 1 & \text { if } x \geq a\end{cases}
$$

The singularity function with $n=-1$ is the Dirac delta function which can be viewed as the derivative of a Heaviside function defined as

$$
\langle x-a\rangle^{-1}= \begin{cases}0 & \text { if } x \neq a \\ \infty & \text { if } x=a .\end{cases}
$$

The singularity function with a negative index with $n>1$ represents symbolically the higher order derivative of a Dirac delta function which becomes meaningful only through distribution. That is,

$$
\int_{-\infty}^{\infty}\langle x-a\rangle^{-n} d x=0
$$

and, for a function $f(x)$ with sufficient regularity,

$$
\int_{-\infty}^{\infty}\langle x-a\rangle^{-n} f(x) d x=\left.(-1)^{n-1}\left(\frac{d^{n-1} f}{d x^{n-1}}\right)\right|_{x=a} .
$$

Let $q(x)$ represent the generalized load, $V(x)$ the shear force, and $M(x)$ the bending moment that is associated with the equilibrium conditions of the beam. The above relations allow the discontinuities to be included in $q(x), V(x)$, and $M(x)$ as follows:

$$
\begin{aligned}
& \frac{d V}{d x}=q(x), \\
& \frac{d M}{d x}=V(x) .
\end{aligned}
$$

The material and the geometrical properties are involved in the deflection equation of the Euler-Bernoulli beam as described by

$$
w^{\prime \prime}(x)=\frac{M(x)}{E I(x)} .
$$

Again, distributional derivatives in deflection will allow the force term on the right-hand side of (9) to include the discontinuities in the moment of inertia and the bending moment. Equation (9) can be extended to include the discontinuities in slope and deflection on its right-hand side in the form of a Dirac delta function and its derivative [6]. Nevertheless, slope and deflection discontinuities will not be considered in this study.

It is noted that the moment of inertia or its reciprocal of a stepped beam can be fully expressed in terms of Heaviside step functions (i.e., zero-order singularity functions). Similarly, the bending moment can also be expressed by singularity functions to count on the discontinuities in loads. The term $M(x) / I(x)$ then involves products of singularity functions of zero or higher order. Consequently, this study focuses on integration of a product of singularity functions of order zero and higher. The key step in the derivation is to span such a product into an algebraic sum of terms, each of which contains only one singularity function. This has to be done with special attention as the definition of the product of singularity functions can be altered by the relationship between function parameters. For example, the outcomes of the following function product depend upon the relationship between $a$ and $b$ :

$$
G_{1}(a, b) \triangleq\langle x-a\rangle^{0}\langle x-b\rangle^{0}= \begin{cases}\langle x-b\rangle^{0} & \text { if } b>a \\ \langle x-a\rangle^{0} & \text { if } a>b .\end{cases}
$$

The general equation for a product between a zero-order singularity function and a higher order one can be found to be

$$
\begin{aligned}
G_{2}(a, b) & \triangleq\langle x-a\rangle^{0}\langle x-b\rangle^{n} \\
& =\left\{\begin{array}{cc}
\left\langle\langle x-b\rangle^{n}\right. & \text { if } b>a \\
\langle\langle x-a\rangle+(a-b)\rangle^{n} & \\
=\sum_{j=0}^{n}\left[C(n, j)\langle x-a\rangle^{n-j}(a-b)^{j}\right] & \text { if } a>b,
\end{array}\right.
\end{aligned}
$$

where $C(n, j)$ is a combination for $n$ choosing $j$. For example, one has the following relation for $n=3$ as

$$
\begin{aligned}
\langle x-a\rangle^{0}\langle x-b\rangle^{3}= & \langle x-a\rangle^{3}+3\langle x-a\rangle^{2}(a-b) \\
& +3\langle x-a\rangle^{1}(a-b)^{2} \\
& +\langle x-a\rangle^{0}(a-b)^{3} .
\end{aligned}
$$

Equation (11) may be extended further to general products of higher order singularity functions. As an example, for the case of $n, m \geq 0$ and $b>a$, one has

$$
\begin{aligned}
G_{3}(a, b) & \equiv\langle x-a\rangle^{m}\langle x-b\rangle^{n} \\
& =\left(\langle x-b\rangle+(b-a)\langle x-b\rangle^{0}\right)^{m}\langle x-b\rangle^{n} \\
& =\sum_{j=0}^{m}\left[C(m, j)\langle x-b\rangle^{n+m-j}(b-a)^{j}\right] .
\end{aligned}
$$

Similar expression can be derived for the cases with $a>b$.

Once it is spanned into terms with single singularity function, $M(x) / I(x)$ can be easily integrated to obtain the 
deflection equation of a beam. Particularly, (9) can be integrated twice to obtain an analytical expression of the beam deflection using (2) along with the expanded algebraic form of $M(x) / I(x)$. For example, integration of $G_{3}$ in (13) yields the following general form for $m, n>0$ and $b>a$ :

$$
\begin{aligned}
\int_{-\infty}^{x} & \langle x-a\rangle^{m}\langle x-b\rangle^{n} d x \\
= & \sum_{j=0}^{m}\left[\frac{C(m, j)}{n+m-j+1}\langle x-b\rangle^{n+m-j+1}(b-a)^{j}\right] .
\end{aligned}
$$

In preparation for sensitivity analysis of the deflection of a stepped beam, the derivative of singularity functions with respect to a design variable is required. The design variables are usually associated with discontinuities presented in the problem such as the location of a support point or the location of discontinuity in the moment of inertia. The differentiation of singularity functions may generate Dirac delta functions. For example, the derivatives of $G_{1}$ and $G_{2}$ with respect to $a$ and $b$ can be obtained as

$$
\begin{gathered}
\frac{d G_{1}}{d a}= \begin{cases}0 & \text { if } b>a \\
-\langle x-a\rangle^{-1} & \text { if } a>b,\end{cases} \\
\frac{d G_{1}}{d b}= \begin{cases}-\langle x-b\rangle^{-1} & \text { if } b>a \\
0 & \text { if } a>b,\end{cases} \\
\frac{d G_{2}}{d a}= \begin{cases}0 & \text { if } b>a \\
\sum_{j=1}^{n}\left\{\begin{array}{l}
C(n, j) \\
\times\left[-(n-j)\langle x-a\rangle^{n-j-1}(a-b)^{j}\right.
\end{array}\right. & \text { if } a>b, \\
\left.\left.+j\langle x-a\rangle^{n-j}(a-b)^{j-1}\right]\right\} & \text { if } b>a \\
\frac{d G_{2}}{d b}=\left\{\begin{array}{l}
n\langle x-b\rangle^{n-1} \\
\sum_{j=1}^{n}\left\{C(n, j)\left[-j(a-b)^{j-1}\right]\right\}
\end{array}\right. & \text { if } a>b .\end{cases}
\end{gathered}
$$

It should be noted that the product of singularity functions that involves a Dirac delta function requires distribution for a meaningful definition.

\section{Static Analysis of Stepped Beams}

Three example stepped beams are used as a vehicle to facilitate discussion on derivation and verification. The detailed derivation for the deflection of a simply supported beam is presented in Section 3.1. With minor modification, the derivation presented in Section 3.1 can be readily extended for the deflections of stepped beams with intermediate supports. The process is briefly discussed in Section 3.2 for an intermediate roller support and in Section 3.3 for a linear spring support. The numerical results of the deflections of these example beams are presented in Section 3.4.

3.1. Simply Supported Stepped Beam. The first example is a simply supported stepped beam as shown in Figure 1. This beam is subjected to two point loads, $F_{1}$ and $F_{2}$, applied at $a_{1}$ and $a_{2}$, respectively. The loads, $F_{1}$ and $F_{2}$, are associated with the dead weights of the gears mounted on the shaft. The beam also experiences discontinuities in the moment of inertia at $b_{1}$ and $b_{2}$. Let $I_{1}$ and $I_{2}$ be the associated moments of inertia, and set $a_{1}<b_{1}<a_{2}$ and $a_{2}<b_{2}<l$.

The equation for the distribution load, $q(x)$, is given below, based upon the free body diagram of the beam:

$$
\begin{aligned}
q(x)= & R_{1}\langle x\rangle^{-1}-F_{1}\left\langle x-a_{1}\right\rangle^{-1} \\
& -F_{2}\left\langle x-a_{2}\right\rangle^{-1}+R_{2}\langle x-l\rangle^{-1} .
\end{aligned}
$$

Note that the reactions forces $R_{1}$ and $R_{2}$ at the supports are included as part of the distribution forces. Using (7), the integration of $q(x)$ from $-\infty$ to $x$ yields the shear force $V(x)$ as

$$
V(x)=R_{1}\langle x\rangle^{0}-F_{1}\left\langle x-a_{1}\right\rangle^{0}-F_{2}\left\langle x-a_{2}\right\rangle^{0}+R_{2}\langle x-l\rangle^{0} .
$$

Similarly, using (8), the bending moment $M(x)$ is obtained by integrating $V(x)$ as

$$
M(x)=R_{1}\langle x\rangle^{1}-F_{1}\left\langle x-a_{1}\right\rangle^{1}-F_{2}\left\langle x-a_{2}\right\rangle^{1}+R_{2}\langle x-l\rangle^{1} .
$$

On the other hand, the distribution of the reciprocal of the moment of inertia can be obtained by

$$
\begin{aligned}
\frac{1}{I(x)}= & \frac{1}{I_{1}}\langle x\rangle^{0}-\left(\frac{1}{I_{1}}-\frac{1}{I_{2}}\right) \\
& \times\left\langle x-b_{1}\right\rangle^{0}-\left(\frac{1}{I_{2}}-\frac{1}{I_{1}}\right)\left\langle x-b_{2}\right\rangle^{0}
\end{aligned}
$$

To maintain an equilibrium of the beam, the shear force $V(x)$ and the bending moment $M(x)$ should be zero right after the right end of the beam; that is, $V(l)=M(l)=0$. Thus, one has

$$
\begin{gathered}
0=R_{1}-F_{1}-F_{2}+R_{2}, \\
0=R_{1} l-F_{1}\left(l-a_{1}\right)-F_{2}\left(l-a_{2}\right)
\end{gathered}
$$

which can be solved for $R_{1}$ and $R_{2}$ as

$$
\begin{gathered}
R_{1}=\frac{F_{1}\left(l-a_{1}\right)+F_{2}\left(l-a_{2}\right)}{l}, \\
R_{2}=\frac{F_{1} a_{1}+F_{2} a_{2}}{l} .
\end{gathered}
$$




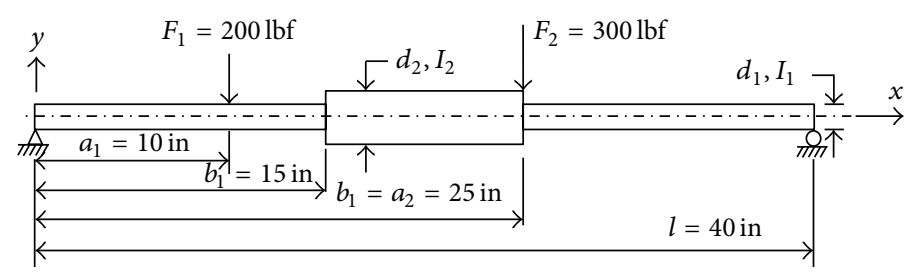

FIGURE 1: A simply supported stepped beam.

The analytical expression of $(M / E I)(x)$ can be simplified by repeatedly using (13) as follows:

$$
\begin{aligned}
\frac{M}{E I}(x)= & \frac{R_{1}}{I_{1}}\langle x\rangle^{1}-\frac{F_{1}}{I_{1}}\left\langle x-a_{1}\right\rangle^{1} \\
& -\left[\left(\frac{R_{1}}{I_{1}}-\frac{R_{1}}{I_{2}}\right) b_{1}-\left(\frac{F_{1}}{I_{1}}-\frac{F_{1}}{I_{2}}\right)\left(b_{1}-a_{1}\right)\right] \\
& \times\left\langle x-b_{1}\right\rangle^{0}-\left[\left(\frac{R_{1}}{I_{1}}-\frac{R_{1}}{I_{2}}\right)-\left(\frac{F_{1}}{I_{1}}-\frac{F_{1}}{I_{2}}\right)\right] \\
& \times\left\langle x-b_{1}\right\rangle^{1}-\left[\left(\frac{R_{1}}{I_{2}}-\frac{R_{1}}{I_{1}}\right) b_{2}\right. \\
& \times\left\langle x-b_{2}\right\rangle^{0}-\left[\left(\frac{R_{1}}{I_{2}}-\frac{R_{1}}{I_{1}}\right)-\left(\frac{F_{1}}{I_{1}}\right)\left(b_{2}-a_{1}\right)\right] \\
& \left.\times\left\langle x-b_{2}\right\rangle^{1}+\left(\frac{F_{2}}{I_{1}}\right)+\frac{F_{2}}{I_{1}}\right] \\
&
\end{aligned}
$$

The deflection curve is then obtained by integrating the above expression twice as, according to (9),

$$
\begin{aligned}
& E w(x)=\iint_{-\infty}^{x} \frac{M(x)}{I(x)} d x d x+C_{1} x+C_{2} \\
& =\frac{R_{1}}{6 I_{1}}\langle x\rangle^{3}-\frac{F_{1}}{6 I_{1}}\left\langle x-a_{1}\right\rangle^{3} \\
& -\frac{1}{2}\left[\left(\frac{R_{1}}{I_{1}}-\frac{R_{1}}{I_{2}}\right) b_{1}-\left(\frac{F_{1}}{I_{1}}-\frac{F_{1}}{I_{2}}\right)\left(b_{1}-a_{1}\right)\right] \\
& \times\left\langle x-b_{1}\right\rangle^{2}-\frac{1}{6}\left[\left(\frac{R_{1}}{I_{1}}-\frac{R_{1}}{I_{2}}\right)-\left(\frac{F_{1}}{I_{1}}-\frac{F_{1}}{I_{2}}\right)\right] \\
& \times\left\langle x-b_{1}\right\rangle^{3}-\frac{1}{2}\left[\left(\frac{R_{1}}{I_{2}}-\frac{R_{1}}{I_{1}}\right) b_{2}\right. \\
& \left.-\left(\frac{F_{1}}{I_{2}}-\frac{F_{1}}{I_{1}}\right)\left(b_{2}-a_{1}\right)\right] \\
& \times\left\langle x-b_{2}\right\rangle^{2}-\frac{1}{6}\left[\left(\frac{R_{1}}{I_{2}}-\frac{R_{1}}{I_{1}}\right)-\left(\frac{F_{1}}{I_{2}}-\frac{F_{1}}{I_{1}}\right)+\frac{F_{2}}{I_{1}}\right] \\
& \times\left\langle x-b_{2}\right\rangle^{3}+\frac{1}{6}\left(\frac{R_{2}}{I_{1}}\right)\langle x-l\rangle^{3}+C_{1} x+C_{2} .
\end{aligned}
$$

The integration constants, $C_{1}$ and $C_{2}$, can be determined based upon the boundary conditions of the given simply supported beam:

$$
w(0)=w(l)=0 .
$$

Thus, one has $C_{2}=0$ and

$$
\begin{aligned}
& C_{1}=-\frac{1}{l}\left\{\frac{R_{1}}{6 I_{1}} l^{3}-\frac{F_{1}}{6 I_{1}}\left(l-a_{1}\right)^{3}\right. \\
&-\frac{1}{2}\left[\left(\frac{R_{1}}{I_{1}}-\frac{R_{1}}{I_{2}}\right) b_{1}-\left(\frac{F_{1}}{I_{1}}-\frac{F_{1}}{I_{2}}\right)\left(b_{1}-a_{1}\right)\right] \\
& \times\left(l-b_{1}\right)^{2}-\frac{1}{6}\left[\left(\frac{R_{1}}{I_{1}}-\frac{R_{1}}{I_{2}}\right)-\left(\frac{F_{1}}{I_{1}}-\frac{F_{1}}{I_{2}}\right)\right] \\
& \times\left(l-b_{1}\right)^{3}-\frac{1}{2}\left[\left(\frac{R_{1}}{I_{2}}-\frac{R_{1}}{I_{1}}\right) b_{2}\right. \\
& \quad \times\left(l-b_{2}\right)^{2}-\frac{1}{6}\left[\left(\frac{F_{1}}{I_{2}}-\frac{F_{1}}{I_{1}}\right)\left(b_{2}-a_{1}\right)\right] \\
&\left.\left.\quad-\left(\frac{F_{1}}{I_{1}}-\frac{F_{1}}{I_{1}}\right)+\frac{F_{2}}{I_{1}}\right]\left(l-b_{2}\right)^{3}\right\} .
\end{aligned}
$$

3.2. Intermediate Roller-Supported Stepped Beam. The derivation for the deflection of the simply supported stepped beam can be easily extended to a stepped beam with an intermediate support. This is accomplished with the help of superposition. An example stepped beam with an intermediate roller support is shown in Figure 2. Let this immediate support be placed at $b_{3}$ for $b_{1}<b_{3}<b_{2}$. The beam becomes statically indeterminate. The deflection of the continuous beam can then be obtained by

$$
v(x)=w(x)+R_{3} \times u(x),
$$

where $v(x)$ is the deflection of the continuous beam, $w(x)$ is the deflection of the simply supported beam, $R_{3}$ is the reaction force at the intermediate support, and $u(x)$ is the deflection of the simply supported beam subjected to a unit force applied at $b_{3}$. That is,

$$
E u^{\prime \prime}(x)=\frac{m(x)}{I(x)},
$$




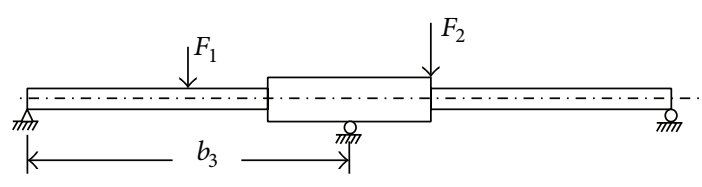

Figure 2: Continuous beam with an intermediate roller support.



FIGURE 3: Continuous beam with an intermediate spring support.

where $m(x)$ is the bending moment given by

$$
m(x)=r_{1}\langle x\rangle^{1}-\left\langle x-b_{3}\right\rangle^{1}+r_{2}\langle x-l\rangle^{1} .
$$

The reciprocal of the moment of inertia, $1 / I(x)$, is given in (19). Note that $r_{1}$ and $r_{2}$ are the reactions at the end supports of the simply supported beam subjected to a unit force. Following the same procedure discussed in Section 3.1, (27) can be integrated twice to obtain the required deflection, $u(x)$.

The reaction force $R_{3}$ at the intermediate support can be calculated based upon the kinematic condition that the deflection at the immediate support must be zero. Thus, one has

$$
R_{3}=-\frac{\left.w\right|_{x=b_{3}}}{\left.u\right|_{x=b_{3}}}
$$

which helps find $v(x)$, based upon (26).

3.3. Intermediate Spring-Supported Stepped Beam. The intermediate roller support located at $b_{3}$ of the stepped beam in Section 3.2 is now replaced by a spring support with a linear spring stiffness $k$. Similar to the intermediate rollersupported beam, (26) can be used to solve a problem of a stepped beam resting on a linear spring. In this case, the reaction force $R_{3}$ at the intermediate support is given by

$$
R_{3}=\frac{\left(\left.k w\right|_{x=b_{3}}\right)}{\left(1-\left.k u\right|_{x=b_{3}}\right)},
$$

where $\left.u\right|_{x=b_{3}}$ represents the displacement at $b_{3}$, where the unit force is placed. The schematic view of the spring-supported beam is shown in Figure 3.

3.4. Numerical Results of Static Analysis. Three stepped beams have been investigated so far: simply supported, an intermediate roller supported, and a linear spring supported. In the numerical study, the length of the beam, $l$, is set to be 40 inches, the weights, $F_{1}$ and $F_{2}, 200 \mathrm{lbs}$ and $300 \mathrm{lbs}$, respectively, and the moments of inertia, $I_{1}$ and $I_{2}, 1.772 \mathrm{in}^{4}$ and $2.505 \mathrm{in}^{4}$, respectively. The locations of the weights, $b_{1}$ and $b_{2}$, are set at $10 \mathrm{in}$. and $25 \mathrm{in}$., respectively, and the
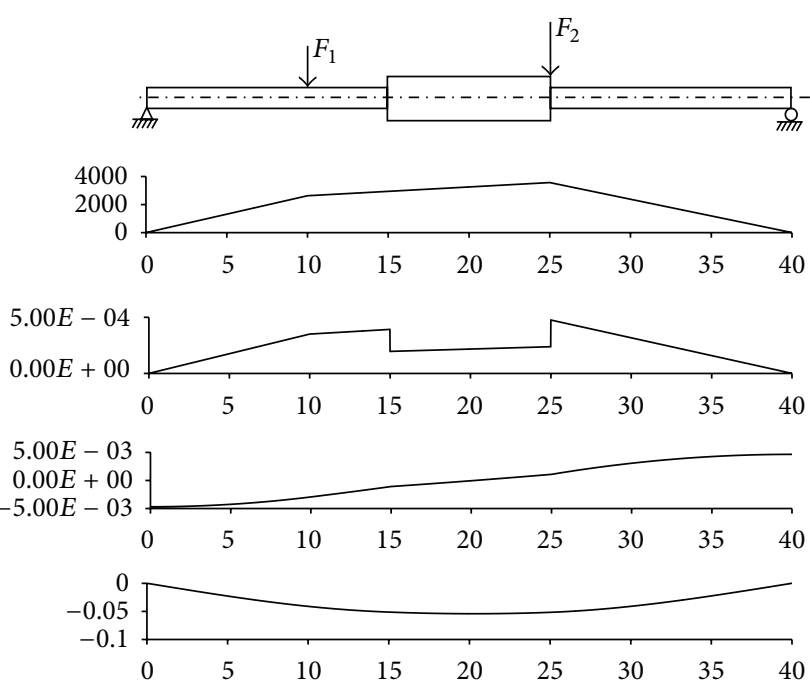

FIGURE 4: The bending moment, the curvature, the slope, and the deflection of the simply supported beam.

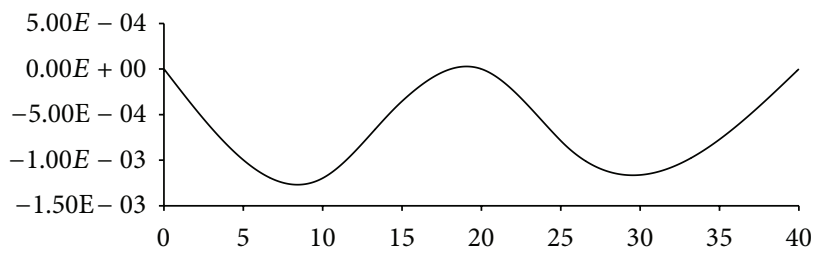

FIGURE 5: Deflection of a continuous beam with an intermediate roller support.

locations for the jumps of flexural rigidity, $a_{1}$ and $a_{2}, 15 \mathrm{in}$. and $25 \mathrm{in}$, respectively. Note that the second weight, $F_{2}$, is placed right at the jump of the flexural rigidity. The material of the beam is assigned with Young's modulus and the Poisson ratio of $30 \times 10^{6} \mathrm{psi}$ and 0.33 , respectively. The density of the material is given by $0.262 \mathrm{lbs} / \mathrm{in}^{3}$. The immediate support and the spring support are placed at the center of the beam; that is, $b_{3}=20 \mathrm{in}$.

The results of static analysis obtained by the singularity functions are first compared with those obtained by the finite element method (FEM). Specifically, the deflection and the slope at 30 inches from the left are reported in Table 1, along with the reactions at the supports. The finite element analysis is done by MD NASTRAN, in which the beam is discretized into 16 beam elements, equally spaced. The results in Table 1 show an excellent agreement between the results of FEM and the method of singularity functions.

The deflection curve of the simply supported stepped beam obtained by the singularity functions is plotted in Figure 4, along with the bending moment, the distribution of $m / I$, and the slope. The schematic view of the simply supported beam is also plotted in the figure to show the locations, where the external forces are applied and the moments of inertia are changed. Similarly, the deflections of the intermediate-supported beam and the spring-supported beam are shown in Figures 5 and 6, respectively. 
TABLE 1: Result comparison for static analysis.

\begin{tabular}{|c|c|c|c|c|c|c|}
\hline \multirow{2}{*}{ Results } & \multicolumn{2}{|c|}{ Simply supported beam } & \multicolumn{2}{|c|}{ Intermediate roller-supported beam } & \multicolumn{2}{|c|}{ Intermediate spring-supported beam } \\
\hline & FEM & Singularity & FEM & Singularity & FEM & Singularity \\
\hline \multicolumn{7}{|l|}{ At $x=30$ in. } \\
\hline Deflection (in) & $-4.109 E-2$ & $-4.109 E-2$ & $-1.161 E-3$ & $-1.161 E-3$ & $-3.872 E-2$ & $-3.872 E-2$ \\
\hline Slope (rad.) & $3.053 E-3$ & $3.053 E-3$ & $1.421 E-5$ & $1.421 E-5$ & $2.873 E-3$ & $2.873 E-3$ \\
\hline \multicolumn{7}{|l|}{ Reactions (lbf) } \\
\hline$R_{1}$ & 262.5 & 262.5 & 47.940 & 47.940 & 249.747 & 249.747 \\
\hline$R_{3}$ & \multicolumn{2}{|c|}{ N.A. } & 429.120 & 429.120 & 25.506 & 25.506 \\
\hline$R_{2}$ & 237.5 & 237.5 & 22.940 & 22.940 & 224.747 & 224.747 \\
\hline
\end{tabular}

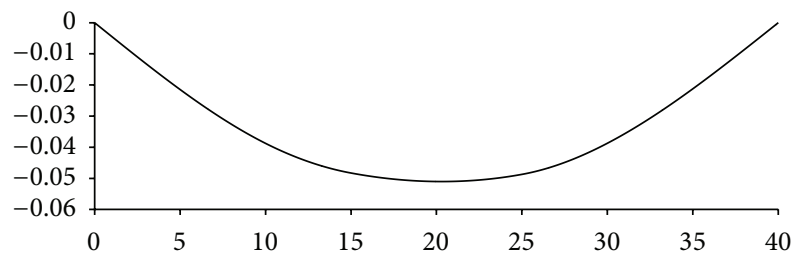

FIGURE 6: Deflection of a continuous beam with an intermediate spring support.

\section{Vibration Analysis of Stepped Beams}

Once the beam deflection functions become available, they can be easily incorporated into a simple procedure to compute the critical speed and its associated mode shapes of a rotating shaft. The procedure relies on the lumped mass model of the stepped beam and uses the beam deflection functions to compute the influence matrix.

The finite element equation for vibration analysis of a shaft is usually represented by

$$
K \mathbf{x}=\lambda M \mathbf{x},
$$

where $K$ and $M$ are the stiffness and the mass matrices of the shaft, respectively. After imposing the kinematic boundary conditions, the stiffness matrix is invertible, and the above equation can be written as

$$
\frac{1}{\lambda} \mathbf{x}=K^{-1} M \mathbf{x}
$$

which is subjected to a normalization condition such as $\mathbf{x}^{T} \mathbf{x}=$ 1. Equation (32) can be conveniently solved by eig in Matlab, once $K^{-1}$ and $M$ are constructed.

Let the shaft be divided into $n$ elements. The mass of each element is lumped at the center of the element. Let $m_{i}$ denote the lumped mass of the $i$ th element. These lumped masses fill the diagonal terms of the diagonal matrix, $M$. A dead weight can be added to the mass matrix by introducing a new mass point that is placed at the location, where the dead weight is applied. The inverse stiffness matrix is the influence matrix $U$ that is the solution of the following matrix equation:

$$
K U=I .
$$

Each column of the matrix $U$, that is, $\mathbf{u}_{i}$, represents a displacement field generated by a force vector that is equal to the $i$ th column of the identifying matrix, $I$. The vector $\mathbf{u}_{i}$ can be conveniently computed based upon the analytical expression of the beam deflection in terms of the singularity functions. Finally, the square, nonsymmetric matrix $K^{-1} M$ is computed as

$$
K^{-1} M=U M=\left[\begin{array}{llllll}
\mathbf{u}_{1} m_{1} & \mathbf{u}_{2} m_{2} & \cdots & \mathbf{u}_{n-1} m_{n-1} & \mathbf{u}_{n} m_{n}
\end{array}\right] .
$$

The $k$ th pair of the eigenvalue and eigenvector, $\left(\lambda_{k}, \mathbf{x}_{k}\right)$, is the solution of (32) or more specifically

$$
\frac{1}{\lambda_{k}} \mathbf{x}_{k}=U M \mathbf{x}_{k}
$$

The eigenvector $\mathbf{x}_{k}$ is the displacement vector evaluated at the points of lumped masses as

$$
\mathbf{x}_{k}=\lambda_{k} K^{-1} M \mathbf{x}_{k}=\lambda_{k} U M \mathbf{x}_{k}
$$

In order to compute $\mathbf{u}_{i}$ in (34), one has to solve the deflection equation in the form of (27) for the stepped beam, subjected to a unit force applied at lumped mass $i$. In this case, the distribution load, the shear force, the bending moment, and the moment of inertia are given, respectively, by

$$
\begin{aligned}
& q(x)=r_{1}\langle x\rangle^{-1}-F\langle x-\xi\rangle^{-1}+r_{2}\langle x-l\rangle^{-1}, \\
& v(x)=r_{1}\langle x\rangle^{0}-F\langle x-\xi\rangle^{0}+r_{2}\langle x-l\rangle^{0} \\
& m(x)=r_{1}\langle x\rangle^{1}-F\langle x-\xi\rangle^{1}+r_{2}\langle x-l\rangle^{1}
\end{aligned}
$$

where $F$ denotes the unit force applied at the $i$ th lumped mass point, $\xi$. The reaction forces, $r_{1}$ and $r_{2}$, are found at the ends of the beam. Here, $r_{1}$ can be easily obtained by

$$
r_{1}=\frac{F \times(l-\xi)}{l}
$$


TABLE 2: Result comparison for vibration analysis.

\begin{tabular}{lcccccc}
\hline \multirow{2}{*}{ Eigenvalues } & \multicolumn{2}{c}{ Simply supported beam } & \multicolumn{2}{c}{ Intermediate roller-supported beam } & \multicolumn{2}{c}{ Intermediate spring-supported beam } \\
& Finite element & Singularity & Finite element & Singularity & Finite element & Singularity \\
\hline$\lambda_{1}$ & $7,816.99$ & $7,780.99$ & $97,610.8$ & $97,155.06$ & $8.3009 E 3$ & $8.2629 E 3$ \\
$\lambda_{2}$ & $97,770.24$ & $97,313.60$ & $402,356.6$ & $400,605.03$ & $9.7771 E 4$ & $9.7314 E 4$ \\
\hline
\end{tabular}

It should be noted that the equation of the moment inertia used here is given by (19), which is the same as that derived for the static analysis. Therefore, $m / I$ can be represented by

$$
\begin{aligned}
\frac{m}{I}= & \frac{r_{1}}{I_{1}}\langle x\rangle^{1}-b_{1}\left(\frac{r_{1}}{I_{1}}-\frac{r_{1}}{I_{2}}\right)\left\langle x-b_{1}\right\rangle^{0} \\
& -\left(\frac{r_{1}}{I_{1}}-\frac{r_{1}}{I_{2}}\right)\left\langle x-b_{1}\right\rangle^{1}-b_{2}\left(\frac{r_{1}}{I_{2}}-\frac{r_{1}}{I_{1}}\right)\left\langle x-b_{2}\right\rangle^{0} \\
& -\left(\frac{r_{1}}{I_{2}}-\frac{r_{1}}{I_{1}}\right)\left\langle x-b_{2}\right\rangle^{1}-F\langle x-\xi\rangle^{1} \\
& \times\left\{\frac{1}{I_{1}}\langle x\rangle^{0}-\left(\frac{1}{I_{1}}-\frac{1}{I_{2}}\right)\left\langle x-b_{1}\right\rangle^{0}\right. \\
& \left.\quad-\left(\frac{1}{I_{2}}-\frac{1}{I_{1}}\right)\left\langle x-b_{2}\right\rangle^{0}\right\} .
\end{aligned}
$$

Setting $\xi$ to be the location of a specific lumped mass, (39) can be integrated twice to obtain the required deflection, $\mathbf{u}_{i}$, according to the procedure discussed in Section 3.1. Once $\mathbf{u}_{i}$ is established, UM can be formed based upon (34). Note that the matrix $U M$ has to be augmented to count for the deflections produced by the external forces, $F_{1}$ and $F_{2}$. The latter can be done, again, following the same procedure as presented in Section 3.1.

The above procedure can be extended for vibration analysis of a continuous stepped beam. In this case, the beam deflections in matrix $U$ require modifications to count for the intermediate supports. Such modifications have been detailed in Sections 3.2 and 3.3.

Numerical exercises are conducted hereafter to verify the solution procedure presented here. The beam studied in Section 3.4 will be repeated here for vibration analysis. The natural frequencies of a stepped beam can be used to investigate the resonance of a rotatory shaft. Two additional weights, $F_{1}$ and $F_{2}$, are added here to the beam to model the gears mounted on the shaft. The stepped beam is divided into 16 elements: 6 on the left thin section, 4 in the middle thick section, and 6 on the right thin section. With additional forces, $F_{1}$ and $F_{2}$, the matrix $U M$ becomes of the size $18 \times 18$. Table 2 shows the comparison of the first two eigenvalues of three different stepped beams using the finite element method and the method of singularity functions. The results show an excellent agreement. The finite element results are obtained from the commercially rated MD NASTRAN, based upon the 16 beam element model.

Equation (36) can be extended to obtain the analytical expression of the eigenfunction, $w_{k}(x)$, associated with the eigenvector $\mathbf{x}_{k}$ at any point $x$ as

$$
w_{k}(x)=\lambda_{k} U M \mathbf{x}_{k} .
$$
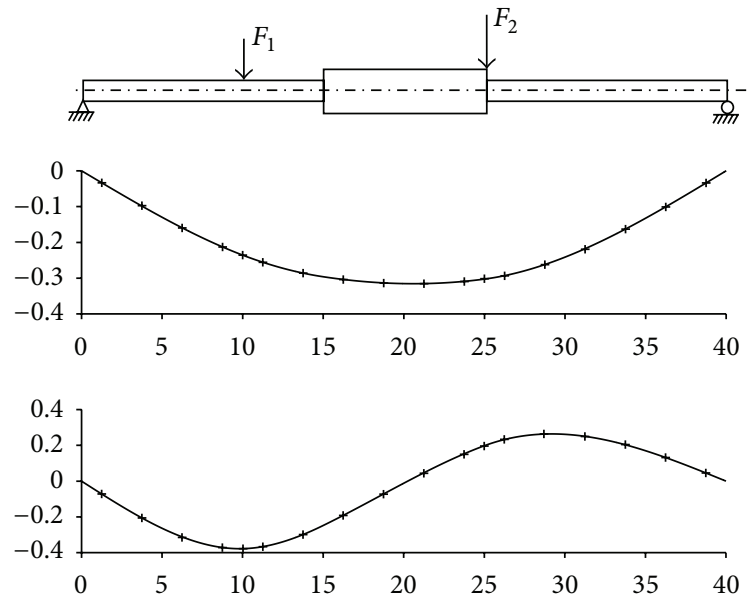

FIGURE 7: The first two vibration modes for simply supported beam.

However, UM, in (40), no longer represents a matrix but a vector made of a collection of beam deflections at any point $x$. These static deflection functions are generated by a set of unit forces applied at each of the lumped mass points, which are already available. Particularly, (40) can be rewritten as

$$
\begin{aligned}
& w_{k}(x)=\lambda_{k} U M \mathbf{x}_{k} \\
& =\lambda_{k}\left[\begin{array}{lll}
u_{1}(x) m_{1} & u_{2}(x) m_{2} & \cdots
\end{array}\right. \\
& \left.u_{n-1}(x) m_{n-1} \quad u_{n}(x) m_{n}\right] \mathbf{x}_{k} \\
& =\lambda_{k} \sum_{j=1}^{n}\left\{u_{j}(x) m_{j} x_{k j}\right\} \text {, }
\end{aligned}
$$

where $u_{j}(x)$ is the deflection equation of the stepped beam subjected to a unit force applied at the lumped mass point $j$, whereas $x_{k j}$ is the $j$ th component of the $k$ th eigenvector, $\mathbf{x}_{k}$.

The first two eigenfunctions resulted from (41) for $k=$ 1 and 2 are shown in Figure 7. The plots are generated by evaluating the eigenfunctions of (41) at 4,000 equally spaced data points. The symbols "+" in the figure mark the locations of lumped masses and force application points.

\section{Design Sensitivity Analysis}

The sensitivity analysis aims to find an efficient means to compute the derivative of structural responses with respect to design variables. The sensitivity analysis has found many 
applications in gradient based design optimization, trade-off design, reliability analysis, and so forth. The design sensitivity analyses of static deflection and eigenvalues of a stepped beam are investigated in this section. The static deflection and eigenvalues of concern are expressed in terms of singularity functions. The sensitivity analysis is performed by using the direct differentiation method. The design variables considered in this study are the locations of rigidity discontinuity and point force.

Since the analytical function of the beam deflection is readily available, it can be directly differentiated with respect to a design variable. Particularly, (23) can be differentiated to obtain the analytical expression of design derivative as

$$
E w_{, b}(x)=\left(\iint_{-\infty}^{x}\left(\frac{M}{I}\right) d x d x\right)_{, b}+C_{1, b} x+C_{2, b}
$$

where the subscript, “, $b$ ”, denotes the design derivative with respect to $b$. It should be mentioned that the differentiation in (42) takes place after integration operation, so as to take advantage of higher function regularity. Furthermore, it is assumed that the location $x$ in (42) is not a function of the design variable.

In the case that the design variable of concern is geometrically related, the concept of material derivative has to be employed to find the design derivative of the deflection $[21,22]$. In this special case, the expression of the deflection of a stepped beam has to be written as $w(x(b), b)$. The implicit relation, $(b)$, implies that the position $x$ of any point in the beam will be changed due to changes in $b$. The total derivative of $w(x(b), b)$ with respect to $b$, denoted by $\bar{w}_{, b}$, can then be evaluated based upon the current state of mesh as

$$
\bar{w}_{, b} \cong \frac{d w}{d b}=w_{, b}+w^{\prime} \times \frac{d x}{d b}
$$

The term $w_{, b}$ is the partial derivative of $w$ with respect to $b$ while $x$ is held constant. This is the term calculated by (42). On the other hand, the term $w^{\prime}$ is the partial derivative of $w$ with respect to $x$ while $b$ is held constant. It is in fact the slope of the beam. The exact expression between $x$ and $b$ is usually defined by the user-specified remeshing process [22], based upon which the derivate, $d x / d b$, can be calculated. Examples are presented hereafter to demonstrate the applications of (42)-(43) for design sensitivity analysis.

5.1. Static Sensitivity Analysis. Only the simply supported stepped beam is investigated here. The design variable is set to be $b_{2}$, where the stepped beam is subjected to rigidity discontinuity and the point load, $F_{2}$. The value of $b_{2}$ is 25 inches.
The derivatives of the deflection curve $E w(x)$ and the constant $C_{1}$ with respect to $b_{2}$ are carried out based upon (23) and (42). They are given by

$$
\begin{aligned}
E w_{, b_{2}}= & \frac{R_{1, b_{2}}}{6 I_{1}}\langle x\rangle^{3}-\frac{1}{2}\left(\frac{R_{1, b_{2}}}{I_{1}}-\frac{R_{1, b_{2}}}{I_{2}}\right) b_{1}\left\langle x-b_{1}\right\rangle^{2} \\
& -\frac{1}{6}\left(\frac{R_{1, b_{2}}}{I_{1}}-\frac{R_{1, b_{2}}}{I_{2}}\right)\left\langle x-b_{1}\right\rangle^{3} \\
& -\frac{1}{2}\left(R_{1, b_{2}} b_{2}+R_{1}-F_{1}\right)\left(\frac{1}{I_{2}}-\frac{1}{I_{1}}\right)\left\langle x-b_{2}\right\rangle^{2} \\
& +\left[R_{1} b_{2}-F_{1}\left(b_{2}-a_{1}\right)\right]\left(\frac{1}{I_{2}}-\frac{1}{I_{1}}\right)\left\langle x-b_{2}\right\rangle^{1} \\
& -\frac{1}{6}\left(\frac{R_{1, b_{2}}}{I_{2}}-\frac{R_{1, b_{2}}}{I_{1}}\right)\left\langle x-b_{2}\right\rangle^{3} \\
& +\frac{1}{2}\left[\left(\frac{R_{1}}{I_{2}}-\frac{R_{1}}{I_{1}}\right)-\left(\frac{F_{1}}{I_{2}}-\frac{F_{1}}{I_{1}}\right)+\frac{F_{2}}{I_{1}}\right] \\
& \times\left\langle x-b_{2}\right\rangle^{2}+C_{1, b_{2}} x,
\end{aligned}
$$

where

$$
\begin{aligned}
C_{1, b_{2}}=-\frac{1}{L}\left\{\frac{R_{1, b_{2}}}{6 I_{1}} L^{3}-\frac{1}{2}\left(\frac{R_{1, b_{2}}}{I_{1}}-\frac{R_{1, b_{2}}}{I_{2}}\right) b_{1}\left(L-b_{1}\right)^{2}\right. \\
-\frac{1}{6}\left(\frac{R_{1, b_{2}}}{I_{1}}-\frac{R_{1, b_{2}}}{I_{2}}\right)\left(L-b_{1}\right)^{3} \\
-\frac{1}{2}\left(R_{1, b_{2}} b_{2}+R_{1}-F_{1}\right)\left(\frac{1}{I_{2}}-\frac{1}{I_{1}}\right)\left(L-b_{2}\right)^{2} \\
+\left[R_{1} b_{2}-F_{1}\left(b_{2}-a_{1}\right)\right]\left(\frac{1}{I_{2}}-\frac{1}{I_{1}}\right)\left(L-b_{2}\right)^{1} \\
-\frac{1}{6}\left(\frac{R_{1, b_{2}}}{I_{2}}-\frac{R_{1, b_{2}}}{I_{1}}\right)\left(L-b_{2}\right)^{3} \\
+\frac{1}{2}\left[\left(\frac{R_{1}}{I_{2}}-\frac{R_{1}}{I_{1}}\right)-\left(\frac{F_{1}}{I_{2}}-\frac{F_{1}}{I_{1}}\right)+\frac{F_{2}}{I_{1}}\right] \\
\times \\
\left.\times\left(L-b_{2}\right)^{2}\right\}, \\
R_{1, b_{2}}=\frac{d R_{1}}{d b_{2}}=-\frac{F_{2}}{L} .
\end{aligned}
$$

As a demonstrative example, the analytical sensitivity derivatives of the deflections and the slopes at points $P$ and $Q$ are calculated based upon (44). The locations of these points are marked in Figure 8(a) at $x_{p}=20$ and $x_{q}=30$ inches. Two different sets of results, $w_{, b_{2}}$ and $\bar{w}_{b b}$, are reported in Table 3 , which are compared with those calculated by the central finite differencing. The location of the rigidity discontinuity $b_{2}$ is considered as the design variable and $\Delta b_{2}$ is set to be 0.25 inch. The analytical value of $w_{, b_{2}}$ is calculated by (44), while 
TABLE 3: Static sensitivity analysis with respect to location of rigidity discontinuity.

\begin{tabular}{|c|c|c|c|c|c|c|}
\hline Sensitivity analysis & Resu & & $\begin{array}{c}\text { Positive } \\
\text { perturbation } \\
\left(b_{2}=25.25^{\prime \prime}\right)\end{array}$ & $\begin{array}{c}\text { Negative } \\
\text { perturbation } \\
\left(b_{2}=24.75^{\prime \prime}\right)\end{array}$ & $\begin{array}{c}\text { Central } \\
\text { difference }\end{array}$ & $\begin{array}{l}\text { Singularity } \\
\text { function }\end{array}$ \\
\hline \multirow{4}{*}{ Without remeshing } & \multirow{2}{*}{ at $x_{p}=20^{\prime \prime}$} & $w_{, b_{2}}$ & $-5.3571 E-2$ & $-5.4899 E-2$ & $2.6561 E-3$ & $2.6563 E-3$ \\
\hline & & $w_{, b_{2}}^{\prime}$ & $-5.1222 E-5$ & $-7.0228 E-5$ & $3.8012 E-5$ & $3.8021 E-5$ \\
\hline & \multirow{2}{*}{ at $x_{q}=30^{\prime \prime}$} & $w_{, b_{2}}$ & $-4.0688 E-2$ & $-4.1488 E-2$ & $1.5989 E-3$ & $1.5990 E-3$ \\
\hline & & $w_{, b_{2}}^{\prime}$ & $3.0050 E-3$ & $3.1016 E-3$ & $-1.9322 E-4$ & $-1.9323 E-4$ \\
\hline \multirow{4}{*}{ With remeshing } & \multirow{2}{*}{ at $x_{p}=20^{\prime \prime}$} & $w_{, b_{2}}$ & $-5.3553 E-2$ & $-5.4865 E-2$ & $2.6231 E-3$ & $2.6259 E-3$ \\
\hline & & $w_{, b_{2}}^{\prime}$ & $-2.4383 E-5$ & $-9.7459 E-5$ & $1.4615 E-4$ & $1.4625 E-4$ \\
\hline & \multirow{2}{*}{ at $x_{q}=30^{\prime \prime}$} & $w_{, b_{2}}$ & $-4.0164 E-2$ & $-4.1980 E-2$ & $3.6325 E-3$ & $3.6359 E-3$ \\
\hline & & $w_{b_{2}}^{\prime}$ & $3.0573 E-3$ & $3.0483 E-3$ & $1.7916 E-5$ & $1.7988 E-5$ \\
\hline
\end{tabular}

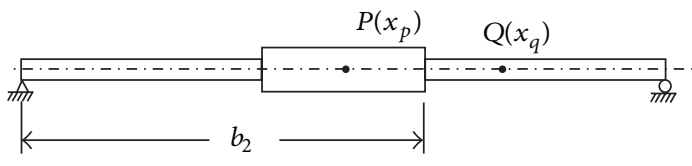

(a)

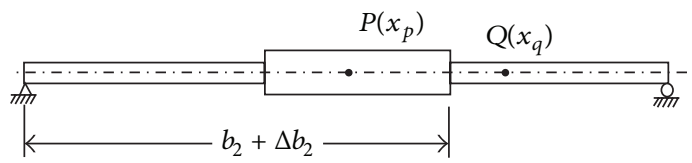

(b)

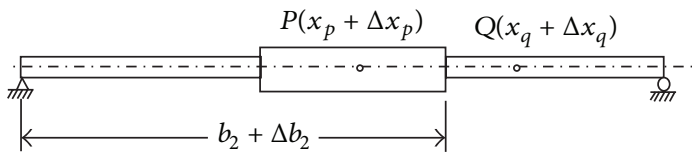

(c)

FIGURE 8: Locations of points $P$ and $Q$ in the beams before and after perturbation in $b_{2}$.

its numerical value is by the following central differencing equation:

$w_{, b_{2}}\left(x, b_{2}\right)=\frac{\partial w\left(x, b_{2}\right)}{\partial b_{2}} \approx \frac{w\left(x, b_{2}+\Delta b_{2}\right)-w\left(x, b_{2}-\Delta b_{2}\right)}{2 \Delta b_{2}}$

Note that (46) is valid only when the perturbation $\Delta b_{2}$ is imposed without involving any remeshing process. As shown in Figure 8(b), the positions of points $P$ and $Q$ remain unchanged before and after $\Delta b_{2}$ being imposed. The upper part of the table shows that the analytical and the finite differencing results are in an excellent agreement. The derivative of the deflection, $w_{, b_{2}}\left(x, b_{2}\right)$, for the entire beam is plotted in Figure 9, which is, once again, matched very well with that obtained by central differencing. Note that the discontinuity in slope occurs at $x=25$ inches in Figure 9, where $b_{2}$ is located.

Next, if a remeshing process is introduced in the perturbed beam to cope with the change in $b_{2}$, the total derivative of $\bar{w}_{, b}(x)$ given by (43) has to be employed for sensitivity

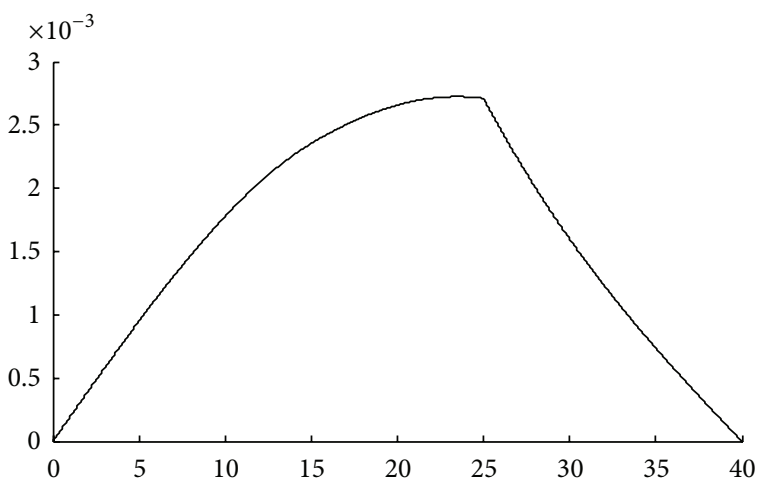

FIGURE 9: Static sensitivity of simply supported beam deflection with respect to $b_{2}$ (without remeshing).

analysis. In this case, the corresponding finite differencing equation becomes

$$
\begin{aligned}
& \bar{w}_{, b_{2}}\left(x, b_{2}\right) \\
& =\frac{d w\left(x\left(b_{2}\right), b_{2}\right)}{d b_{2}} \\
& \quad \approx \frac{w\left(x\left(b_{2}+\Delta b_{2}\right), b_{2}+\Delta b_{2}\right)-w\left(x\left(b_{2}-\Delta b_{2}\right), b_{2}-\Delta b_{2}\right)}{2 \Delta b_{2}} .
\end{aligned}
$$

As an example, let positions of $P$ and $Q$ be changed to $x_{p}+\Delta x_{p}$ and $x_{q}+\Delta x_{q}$, respectively, in the perturbed beam, as shown in Figure 8(c). The changes $\Delta x_{p}$ and $\Delta x_{q}$ can be conveniently determined based upon the user specified remeshing process. In this example, they are specified as

$$
\begin{aligned}
\Delta x_{p} & =\frac{x_{p}-b_{1}}{b_{2}-b_{1}} \Delta b_{2}, \\
\Delta x_{q} & =\frac{l-x_{q}}{l-b_{2}} \Delta b_{2} .
\end{aligned}
$$


TABLE 4: Eigenvalue sensitivity analysis with respect to location of rigidity discontinuity, $b_{2}$.

\begin{tabular}{lcccc}
\hline Eigenvalues & Positive perturbation $\left(b_{2}=25.25\right.$ in. $)$ & Negative perturbation $\left(b_{2}=24.75\right.$ in. $)$ & Central difference & Singularity function \\
\hline$\lambda_{1}$ & $7.9174 E 3$ & $7.6664 E 3$ & $5.0189 E 2$ & $5.0174 E 2$ \\
$\lambda_{2}$ & $9.5865 E 4$ & $9.9055 E 4$ & $-6.3783 E 3$ & $-6.3754 E 3$ \\
\hline
\end{tabular}

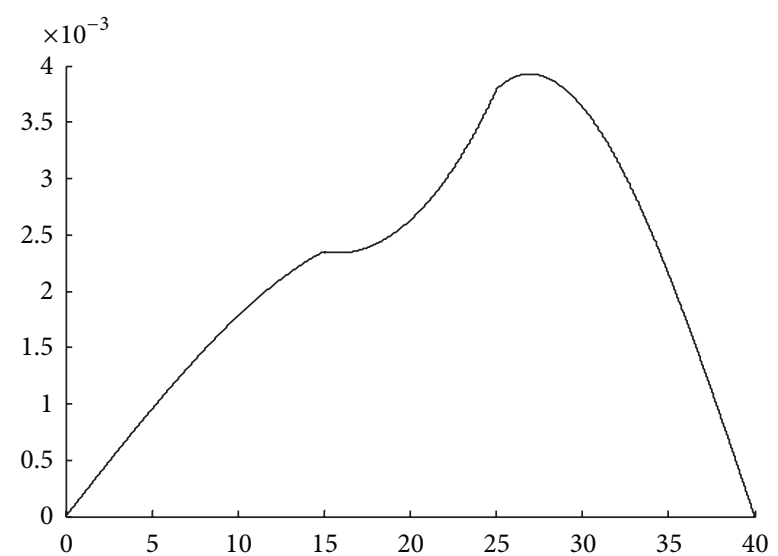

Figure 10: Total static sensitivity of simply supported beam deflection with respect to $b_{2}$ (with remeshing).

Such a remeshing process produces the following mesh derivative which is required to evaluate $\bar{w}_{, b_{2}}(x)$ by (43) as

$$
\frac{d x}{d b_{2}}= \begin{cases}0 & 0 \leq x \leq b_{1} \\ \frac{x-b_{1}}{b_{2}-b_{1}} & b_{1} \leq x \leq b_{2} \\ \frac{l-x}{l-b_{2}} & b_{2} \leq x \leq l .\end{cases}
$$

The total static sensitivity of the beam deflections and the slopes at points $P$ and $Q$ can then be calculated by (43). The lower part of Table 3 shows that the total derivatives calculated analytically matched very well with those by finite differencing. The total derivative of the deflection for the entire beam, $\bar{w}_{, b_{2}}(x)$, is plotted in Figure 10, which again is the same as that obtained by finite differencing.

5.2. Eigenvalue Sensitivity Analysis. The eigenvalue sensitivity analysis of the simply supported beam example is investigated here. The location of the rigidity discontinuity, $b_{2}$, is considered as the design variable. The derivation is based upon the nonsymmetric eigenvalue equation, (35). Differentiating the $k$ th pair of eigenvalue and eigenvector that satisfy (35) with respect to the design variable, $b_{2}$, leads to

$$
\frac{1}{\lambda_{k}} \frac{d \mathbf{x}_{k}}{d b_{2}}-\frac{\mathbf{x}_{k}}{\lambda_{k}^{2}} \frac{d \lambda_{k}}{d b_{2}}=\frac{d(U M)}{d b_{2}} \mathbf{x}_{k}+U M \frac{d \mathbf{x}_{k}}{d b_{2}} .
$$

Premultiplying the left eigenvector of (35), $\mathbf{y}_{k}^{T}$, to (51) yields the desired eigenvalue sensitivity as

$$
\frac{d \lambda_{k}}{d b_{2}}=-\frac{\lambda_{k}^{2}}{\mathbf{y}_{k}^{T} \mathbf{x}_{k}} \mathbf{y}_{k}^{T} \frac{d(U M)}{d b_{2}} \mathbf{x}_{k}
$$

Note that the left eigenvector, $\mathbf{y}_{k}^{T}$, is the solution of the following eigenvalue problem:

$$
\frac{1}{\lambda_{k}} \mathbf{y}_{k}=(U M)^{T} \mathbf{y}_{k} .
$$

Calculation of the derivative formula, $d(U M) / d b_{2}=$ $\left(d U / d b_{2}\right) M+U\left(d M / d b_{2}\right)$, in (52) requires special attention, particularly if both the locations and the values of the lumped masses are functions of $b_{2}$. Since each column of $U$ is evaluated at the lumped mass points, their derivatives with respect to $b_{2}$ in this case have to be computed by (43) as a total derivative.

The numerical results of the eigenvalue sensitivities of the first and the second eigenvalues of the example simply supported beam are summarized in Table 4 . The results of analytical derivatives are compared with those obtained by finite differencing in this table. Table 4 demonstrates that the results are in an excellent agreement.

Numerical results reported in Table 4 are done with the example beam divided into 16 segments. Six segments are equally spaced in each of the left and the right thin sections of the beam, while four equally spaced segments are made in the middle thick section. With $b_{2}$ being set at 25 inches, the length of every segment in the example beam is 2.5 inches. The central differencing method in Table 4 imposes \pm 0.25 as the perturbation of $b_{2}$. Once $b_{2}$ is changed, however, the length of each segment is changed, which in turn changes the values and the locations of the lumped masses. Specifically, the remeshing equation of (48) can be employed here to move the location, $x_{i}$, of the $i$ th lumped mass in the middle thick section. As such, the location $x_{i}$ is defined by

$$
x_{i}=b_{1}+\frac{(2 i-1)\left(b_{2}-b_{1}\right)}{2 n_{1}} .
$$

Accordingly, the value of the $i$ th lumped mass will be changed as

$$
m_{i}=\rho A \frac{\left(b_{2}-b_{1}\right)}{n_{1}} .
$$

The notation $n_{1}$ in both equations is the number of segments in the middle thick section and $1 \leq i \leq n_{1}$. In this example, $n_{1}=4$. The above equations give

$$
\frac{d x_{i}}{d b_{2}}=\frac{(2 i-1)}{2 n_{1}}
$$

which will be used in (43) to compute $\bar{w}_{, b_{2}}$ at $x_{i}$. Similarly, the remeshing process of (49) can be employed here to relocate the lumped mass points in the right thin section. The assembly of $\bar{w}_{, b_{2}}$ at all lumped mass points then forms 



FIGURE 11: Total derivatives of the beam deflections for a unit load applied at $x=24.75$ and $x=25.25$ inches.

the corresponding column in $d \mathbf{U} / d b_{2}$. Furthermore, the mass derivative is obtained as follows:

$$
\frac{d m_{i}}{d b_{2}}=\frac{\rho A}{2 n_{1}}
$$

which can be used to compute $d M / d b_{2}$.

To further verify (43) for sensitivity analysis, the example beam is discretized into 80 segments in which $n_{1}$ is set to be 20. The total derivatives $\bar{w}_{, b_{2}}$ are plotted in Figure 11 for the beam deflections subjected to a unit load applied at $x=24.75$ and $x=25.25$ inches, respectively. They are matched very well with those obtained by finite differencing (47).

Note that $x\left(b_{2}\right)$ in this example is specified by the process of remeshing the stepped beam according to (48) and (49). Figure 11 reveals that the sensitivity of the beam deflection subjected to a point load applied on the left of the rigidity discontinuity is significantly different from that applied at an adjacent point on the right.

\section{Concluding Remarks}

The stepped beams studied here are subjected to discontinuities in flexural rigidity and applied loads. The derivation of the deflection function of a statically determinate stepped beam is achieved in a two-step process. In the first step, the applied load is formulated and integrated twice to obtain the distribution of bending moment. The moment distribution is then multiplied by the reciprocal of the rigidity distribution to form the right-hand side of a second order differential equation of beam deflection. Many right-hand side terms are made of products of two singularity functions, each of which can be spanned into terms with only one singularity function. As a result, the second order differential equation can be easily integrated twice to obtain the desirable deflection function. The same approach can be extended to find the deflection function of a statically indeterminate stepped beam. This paper demonstrates and verifies such an approach by using examples of an intermediate roller-supported and an intermediate spring-supported beam.
The above solution process is further extended to vibration analysis of stepped beams. This is accomplished based upon the lumped mass approach. The eigenfunction of a stepped beam is, in fact, a linear combination of deflection functions of the beam of concern. Each of such deflection functions results from the beam subjected to a unit force applied at a lumped mass point.

It should be noted that the analytical expressions of the beam deflection presented in this paper are all in terms of singularity functions with order higher than zero. Consequently, they can be conveniently differentiated with respect to any beam-related design variable. In this study, design derivatives of a static deflection and the eigenvalue of a stepped beam with respect to the location of the rigidity discontinuity are derived. Such design derivatives have to be considered in the context of shape design sensitivity. Their numerical values are verified with those of finite differencing.

\section{Conflict of Interests}

The authors declare that there is no conflict of interests regarding the publication of this paper.

\section{References}

[1] W. D. Pilkey, "Some properties and applications of singularity functions based on the theory of distributions," Journal of the Franklin Institute, vol. 277, no. 5, pp. 464-497, 1964.

[2] R. L. Norton, Machine Design: An Integrated Approach, Prentice-Hall, Upper Saddle River, NJ, USA, 1st edition, 1996.

[3] R. G. Budynas and J. K. Nisbett, Shigley's Mechanical Engineering Design, McGraw Hill, New York, NY, USA, 8th edition, 2008.

[4] F. Van Keulen, R. T. Haftka, and N. H. Kim, "Review of options for structural design sensitivity analysis. Part 1: linear systems," Computer Methods in Applied Mechanics and Engineering, vol. 194, no. 30-33, pp. 3213-3243, 2005.

[5] M. Bogomolni, U. Kirsch, and I. Sheinman, "Efficient design sensitivities of structures subjected to dynamic loading," International Journal of Solids and Structures, vol. 43, no. 18-19, pp. 5485-5500, 2006.

[6] G. Falsone, "The use of generalised functions in the discontinuous beam bending differential equations," International Journal of Engineering Education, vol. 18, no. 3, pp. 337-343, 2002.

[7] A. Yavari, S. Sarkani, and E. T. Moyer Jr., "On applications of generalized functions to beam bending problems," International Journal of Solids and Structures, vol. 37, no. 40, pp. 5675-5705, 2000.

[8] A. Yavari, S. Sarkani, and J. N. Reddy, "Generalized solutions of beams with jump discontinuities on elastic foundations," Archive of Applied Mechanics, vol. 71, no. 9, pp. 625-639, 2001.

[9] B. Biondi and S. Caddemi, "Closed form solutions of EulerBernoulli beams with singularities," International Journal of Solids and Structures, vol. 42, no. 9-10, pp. 3027-3044, 2005.

[10] B. Biondi and S. Caddemi, "Euler-Bernoulli beams with multiple singularities in the flexural stiffness," European Journal of Mechanics A, vol. 26, no. 5, pp. 789-809, 2007.

[11] A. Palmeri and A. Cicirello, "Physically-based Dirac's delta functions in the static analysis of multi-cracked Euler-Bernoulli and Timoshenko beams," International Journal of Solids and Structures, vol. 48, no. 14-15, pp. 2184-2195, 2011. 
[12] M. Skrinar, "Computational analysis of multi-stepped beams and beams with linearly-varying heights implementing closedform finite element formulation for multi-cracked beam elements," International Journal of Solids and Structures, vol. 50, no. 14-15, pp. 2527-2541, 2013.

[13] G. Duan and X. Wang, "Free vibration analysis of multiplestepped beams by the discrete singular convolution," Applied Mathematics and Computation, vol. 219, no. 24, pp. 11096-11109, 2013.

[14] S. Naguleswaran, "Natural frequencies, sensitivity and mode shape details of an Euler-Bernoulli beam with one-step change in cross-section and with ends on classical supports," Journal of Sound and Vibration, vol. 252, no. 4, pp. 751-767, 2002.

[15] S. Naguleswaran, "Vibration of an Euler-Bernoulli beam on elastic end supports and with up to three step changes in crosssection," International Journal of Mechanical Sciences, vol. 44, no. 12, pp. 2541-2555, 2002.

[16] M. A. Koplow, A. Bhattacharyya, and B. P. Mann, "Closed form solutions for the dynamic response of Euler-Bernoulli beams with step changes in cross section," Journal of Sound and Vibration, vol. 295, no. 1-2, pp. 214-225, 2006.

[17] S. C. Stanton and B. P. Mann, "On the dynamic response of beams with multiple geometric or material discontinuities," Mechanical Systems and Signal Processing, vol. 24, no. 5, pp. 1409-1419, 2010.

[18] N. Popplewell and D. Chang, "Free vibrations of a complex Euler-Bernoulli beam," Journal of Sound and Vibration, vol. 190, no. 5, pp. 852-856, 1996.

[19] Z. R. Lu, M. Huang, J. K. Liu, W. H. Chen, and W. Y. Liao, "Vibration analysis of multiple-stepped beams with the composite element model," Journal of Sound and Vibration, vol. 322, no. 4-5, pp. 1070-1080, 2009.

[20] U. Lepik, "Optimal design of elastic-plastic stepped beams under dynamic loading," Structural Optimization, vol. 7, no. 12, pp. 87-90, 1994.

[21] J. W. Hou and J. L. Chen, "Shape optimization of elastic hollow bars," Journal of mechanisms, Transmissions, and Automation in Design, vol. 107, no. 1, pp. 100-105, 1985.

[22] J. W. Hou, J. L. Chen, and J. S. Sheen, "A computational method for optimization of structural shapes," AIAA Journal, vol. 24, no. 6, pp. 1005-1012, 1986. 

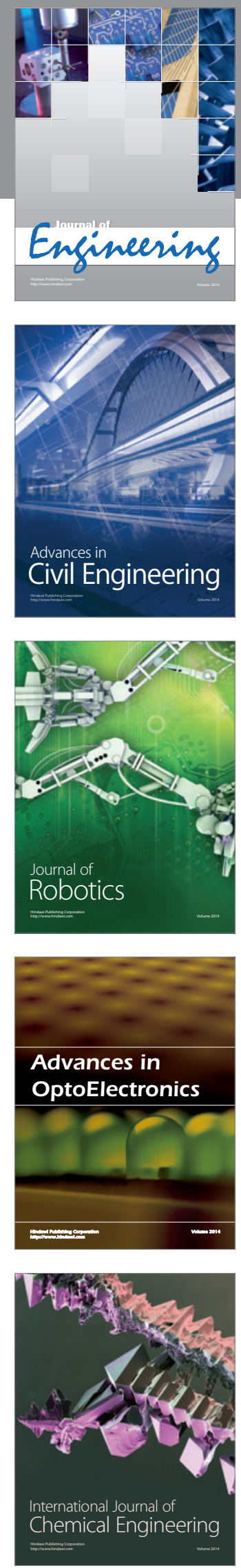

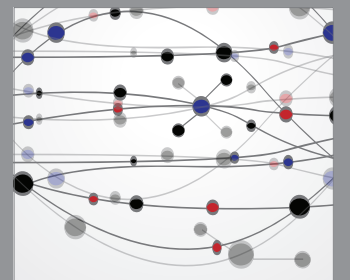

The Scientific World Journal
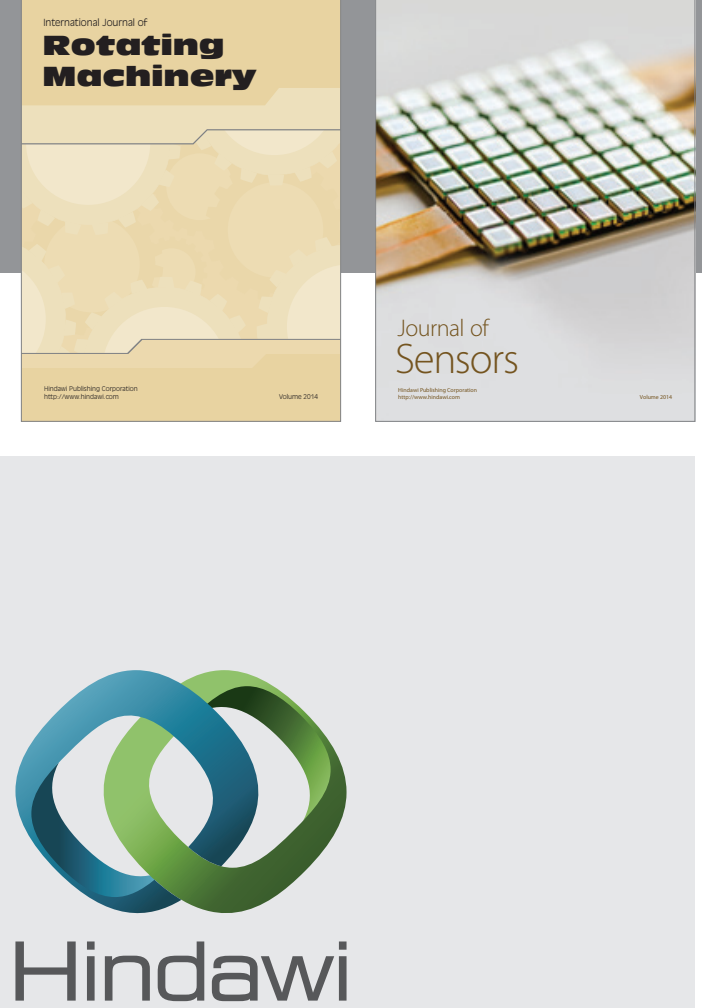

Submit your manuscripts at http://www.hindawi.com

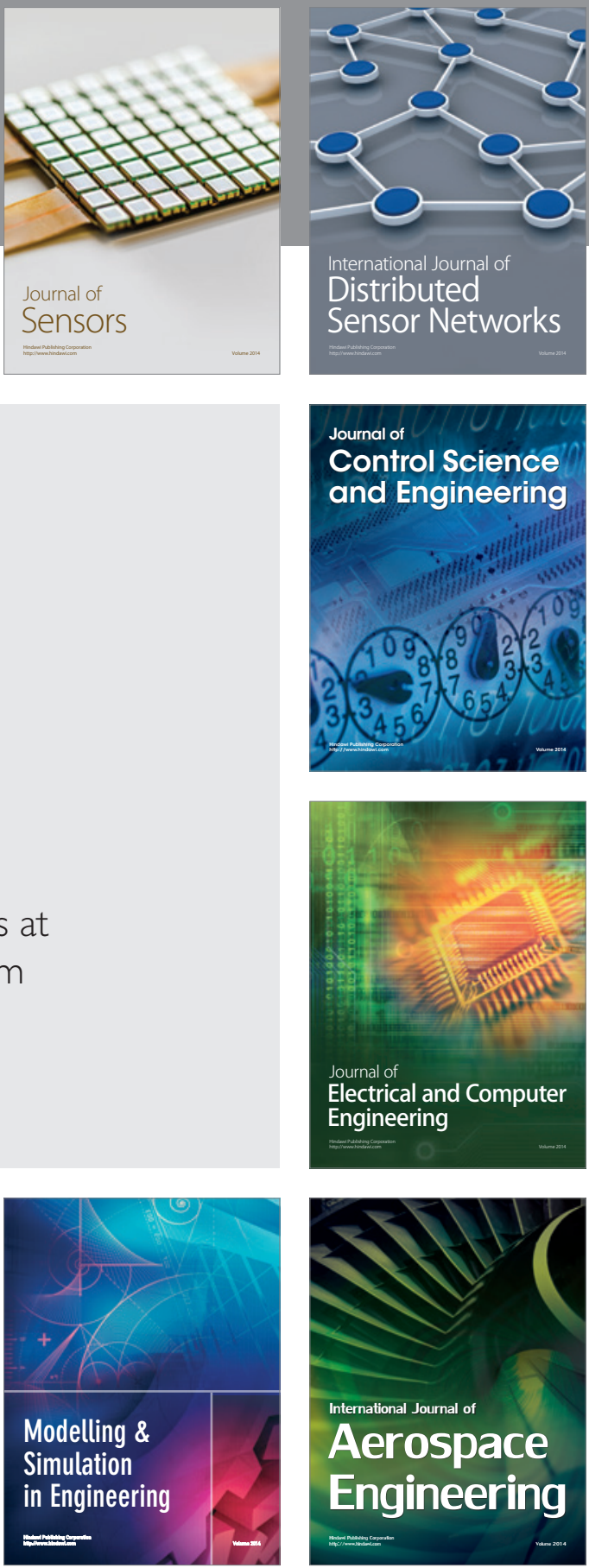

Journal of

Control Science

and Engineering
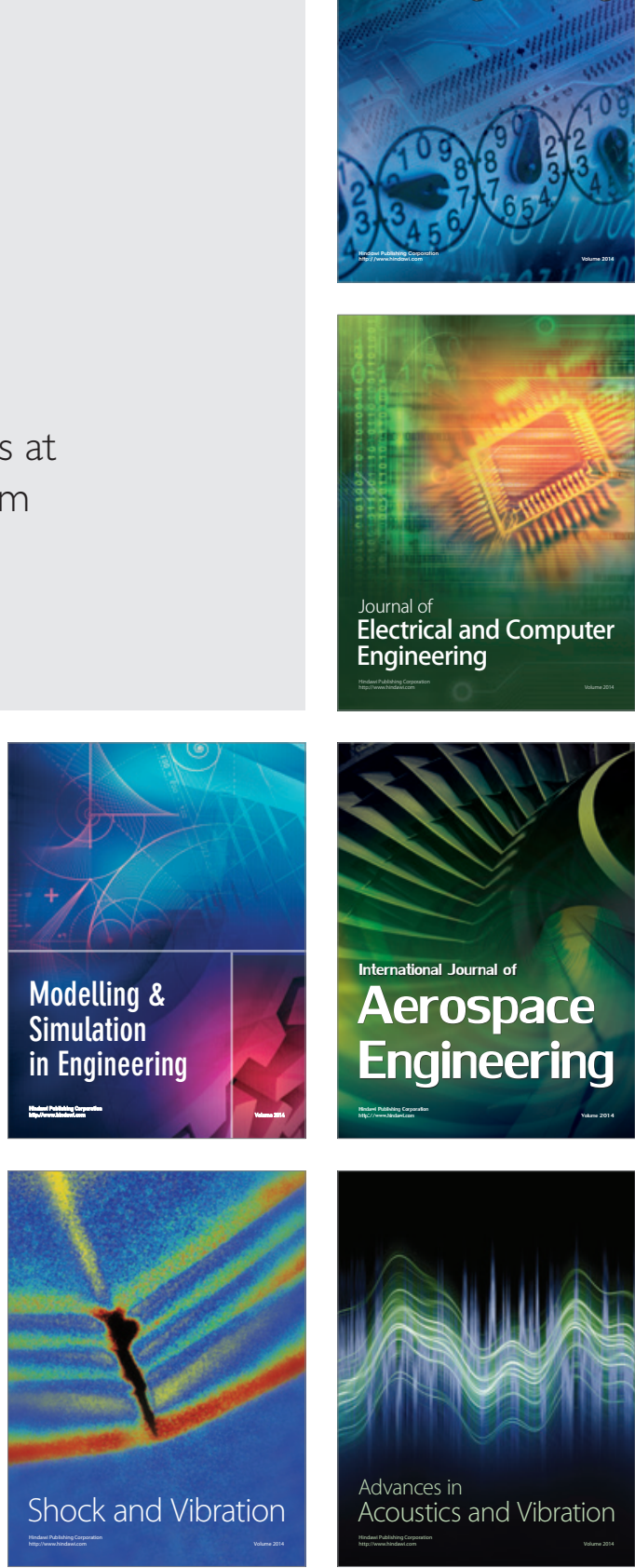